\title{
BMJ Open Being there: protocol for a scoping review of the medical education literature on grief support training for medical professionals
}

\author{
Sophie Soklaridis, ${ }^{1,2,3}$ Genevieve Ferguson, ${ }^{1}$ Sarah Bonato, ${ }^{1}$ Riley Saikaly, ${ }^{1}$ \\ Pamela J Mosher ${ }^{1,2}$
}

To cite: Soklaridis $S$, Ferguson G, Bonato S, et al. Being there: protocol for a scoping review of the medical education literature on grief support training for medical professionals. BMJ Open 2018;8:e022778. doi:10.1136/ bmjopen-2018-022778

- Prepublication history and additional material for this paper are available online. To view these files, please visit the journal online (http://dx.doi. org/10.1136/bmjopen-2017022778).

Received 8 March 2018 Revised 12 September 2018 Accepted 9 November 2018

Check for updates

(C) Author(s) (or their employer(s)) 2018. Re-use permitted under CC BY-NC. No commercial re-use. See rights and permissions. Published by BMJ.

${ }^{1}$ Office of Education, Centre for Addiction and Mental Health, Toronto, Ontario, Canada ${ }^{2}$ Departments of Psychiatry and Family and Community Medicine, University of Toronto Toronto, Ontario, Canada ${ }^{3}$ Cross-Appointed Scientist, Wilson Centre, University Health Network and Faculty of Medicine, University of Toronto, Toronto, Ontario, Canada

Correspondence to

Dr Sophie Soklaridis;

Sophie.Soklaridis@camh.ca

\section{ABSTRACT}

Introduction Medical trainees and professionals do not perceive that they are adequately taught the skills to address issues of grief with their patients. Atypical grief responses can prolong suffering, interrupt normal activities and lead to increased morbidity and mortality. Grief training can help physicians cope with feelings about and responses to suffering, loss and death in a way that improves both physician and patient/family wellness. This scoping review will describe the current landscape of grief training worldwide in medical school and residency and in continuing professional development in the disciplines of paediatrics, family medicine and psychiatry. The ultimate goal is to help physicians support patients experiencing grief.

Methods and analysis The study design has been adapted from Arksey and 0'Malley's review methodology. We will work with an information specialist who will run searches in six multidisciplinary databases. To supplement the search, we will scan the reference lists of included studies. Two levels of screening will take place: a title and abstract review for articles that fit predefined criteria and a full-text review of articles that meet those criteria. To be included in the review, articles must report on grief training for medical residents and professionals in the fields of paediatrics, family medicine and psychiatry. Two investigators will review each article and extract data. Ethics and dissemination Research ethics approval is not required for this review. We plan to share the findings through national and international medical education conferences and to publish the results in a peer-reviewed academic journal. We have the support of several directors of medical education at our institution who are interested in the growing focus on humanism in medical education as a way of decreasing burnout among medical students, residents and faculty.

\section{BACKGROUND}

The loss of a loved one is one of life's most challenging experiences. People have often described it as among the most isolating and intensely emotional periods of their lives. The pain and isolation associated with loss are particularly acute when the loss is untimely or
Strengths and limitations of this study

- The findings of this review will be the first to provide a baseline understanding of grief support training in general and training specific to children and family members who are grieving the loss of a child in undergraduate medical school, residency curricula and continuing professional development.

- This protocol details a rigorous study design informed by an established review methodology by Arksey and 0'Malley, a robust search strategy developed iteratively in consultation with an experienced information specialist and a thoughtful study selection and abstraction process that involves calibration between two independent reviewers.

- The review will not attempt to measure the effectiveness of grief training interventions and will be limited to descriptive narrative analysis.

- Although the review will be robust with respect to the article types and methodologies, the findings will be limited to articles written in English.

- The studies included in the review will not undergo a quality appraisal process.

unexpected, such as the death of a child or, for a young child, the death of a parent or sibling. Family members are at elevated risk of depression and anxiety for close to a decade after the loss of a child. ${ }^{12}$ For children in later childhood, the loss of a parent can result in emotional and behavioural problems, as well as increased vulnerability to psychiatric and medical comorbidities later in life. Preparing a child for the loss and giving them access to support when it happens have been shown to reduce morbidity after bereavement. ${ }^{3}$ Physicians play an important role in supporting their patients to adapt to their loss.

People can have an atypical grief response (eg, delayed grief, complicated or prolonged grief, denial and absent grief), which can prolong suffering, interrupt normal functioning or lead to increased morbidity and 
mortality. ${ }^{45}$ However, the literature on grief and bereavement does not present a uniform empirical definition of grief, including what features constitute an abnormal course of grief. ${ }^{67}$ The Diagnostic and Statistical Manual of Mental Disorders, Fifth Edition (DSM-5) describes the death of a close relative as a stressor, with normative and predictable symptoms. ${ }^{8}$ It also indicates that grief behaviours, including duration and expression of typical bereavement, vary immensely across cultural groups and settings (eg, type of relationship) ${ }^{9}$ Complicating the matter further, there is debate in the medical community about pathologising bereavement and about how we differentiate grief behaviours from symptoms of major depressive disorder, anxiety or post-traumatic stress disorder.

Research has found that medical students, residents and physicians often believe they lack the skills to adequately address grief..$^{10}$ Reviews of the literature note that end-oflife education, predominantly presented within the preclinical curriculum, tends to focus on knowledge rather than attitudes and does not often allow important opportunities for students to reflect on their own experiences of grief. ${ }^{11}$ It has been argued that training should help learners become aware of their own attitudes towards death and caring for patients at the end of life. ${ }^{12}$ Furthermore, systematic analysis of palliative care curricula in medical schools shows that diverse, non-standardised formats are being used to incorporate this subspecialty's principles and evidence-based data into the preclinical years. ${ }^{13}$ Thus, medical students receive widely variable training with respect to knowledge, skills and attitudes in the domains of dying, death and grief.

Certain medical subspecialties, such as family medicine, paediatrics and psychiatry, involve prolonged engagement with patients and family members that can last for several years. As such, these physicians are most likely to experience the loss of a patient and to be involved in the aftercare of grieving family members. Even so, medical education to prepare these physicians for their role in grief support is lacking. A recent study of general practitioners revealed a perceived lack of education around the multifaceted nature of grief. ${ }^{14}$ Respondents reported lacking formal education about grief or having very limited exposure to information about grief during medical school. A study of paediatric residents' knowledge, skills and attitudes about death and dying found that residents feel unprepared to deal with dying patients or grieving family members. ${ }^{15}$ Even in psychiatry, it has been noted that residency education is not preparing psychiatrists to feel competent in managing the complicated issues that arise when a patient dies. ${ }^{16}$ This lack of knowledge and skills seems to continue into clinical practice, with few identifiable opportunities to learn about grief support through continuing professional development.

The task of identifying grief and supporting patients is challenging and is a cause of burnout and emotional distress among physicians. ${ }^{1718}$ Grief training is believed to help physicians cope with feelings about and responses to suffering, loss and death in a way that improves physician wellness. ${ }^{19}$ The importance of providing physicians with the knowledge and skills to offer grief support to patients and families at one of the most critical points in their lives cannot be understated.

We will conduct a scoping review of the scientific literature to understand the extent to which grief support training in general, and training specific to children and family members grieving the loss of a child, are offered in the undergraduate medical school and residency curricula and in continuing professional development. The review depicts the current landscape of grief training worldwide in medical school and residency in the disciplines of paediatrics, family medicine and psychiatry. Ultimately, improved understanding of available grief training, including underlying approaches used to inform this training, will enable both programme development and scholarship in this area. To the best of our knowledge, there are no current data on standardised grief training at Canadian medical schools or residency programmes.

\section{METHODS}

\section{Patient and public involvement}

Patients are not involved in the design of this scoping review study.

\section{Scoping review}

Scoping reviews examine the existing literature to map the extent and range of a field. ${ }^{20}{ }^{21}$ Given the paucity of curricular opportunities to learn about grief during medical school and residency and in continuing professional development, we adapted Arksey and O'Malley's seminal paper on scoping review methodology to systematically review the depth and breadth of the literature in this field as represented by all publications about research and curriculum design. ${ }^{22}$ The proposed review will be conducted in five phases. Although the following sections describe the phases in linear order, there may be overlap as each phase feeds into the next.

\section{Planning and research question}

This scoping review study was developed through the Medical Psychiatry Alliance initiative, a four-institution partnership in conjunction with the Ontario Ministry of Health and Long-Term Care and a donor. The goal of this partnership is to transform the delivery of mental health services for patients with comorbid physical and psychiatric illness. The planning phase of the study included initial collaboration with a scientist (SS) and a psychiatrist/ paediatrician (PJM). An environmental scan of the literature revealed that some academic literature in the field of grief training in medical education does exist, but few studies describe outcomes or impact, and thus a systematic review was not considered. The lack of grief training is particularly concerning in paediatric care because children with medical illnesses face loss on loss-of physical 
capacity or autonomy; of control; of normal cognitive, educational, emotional, social and sexual development; of peer group; of contact with siblings or pets and, for palliative care patients, loss of a sense of future and life. We specifically focused on grief training in paediatrics, family medicine and psychiatry because the results of this scoping review will be used to advocate for changes in the curriculum in these respective departments of which the authors hold faculty appointments. If there are not enough articles on grief training in these three medical disciplines, we plan to broaden the search to include other medical disciplines such as emergency medicine, oncology and obstetrics.

Our study will focus on grief, not depression. There are critical differences between these two conditions. Grief is the biopsychosocial response to the loss of a loved one. ${ }^{23}$ It is often believed to be permanent, although less intrusive and all-consuming over time. Grief is not a form of depression. ${ }^{6}$ Depression is a psychiatric disorder that requires treatment. Although grief may turn a person inward, the person is still able to appreciate the efforts others make to provide support. People with depression, on the other hand, may lack the capacity to care about other people and to understand their good intentions. ${ }^{23}$ It is important that physicians not confound grief and depression because many people who have lost a loved one will turn to family physicians, paediatricians and psychiatrists for support and reassurance, not for treatment. ${ }^{6}$

On receiving funding, we hired a research analyst (GF) and an information specialist (SB) who participated in planning and developing the research question. Our research question was: what is known from the existing academic literature about grief training in medical school, residency programmes and continuing professional development in psychiatry, family medicine and paediatrics? Identifying past and current grief curriculum and training opportunities will allow us to determine gaps in knowledge and to develop recommendations for the way clinical teaching about grief is delivered to current and future health professionals.

\section{Information sources}

An experienced information specialist will search the following electronic databases from inception onwards: MEDLINE (Ovid); Embase (Ovid); PsycINFO (Ovid); all EBM Reviews-Cochrane DSR, ACP Journal Club, DARE, CCTR, CMR, HTA and NHSEED (Ovid); ERIC (Ovid); CINAHL; LISA (ProQuest); Philosopher's Index (ProQuest); Scopus; Social Sciences Abstracts (Wilson); and Web of Science. To avoid publication bias and to improve the overall reach and spread of this scoping review, we will also perform a targeted streamlined grey literature search (see online supplementary file 1).

The search strategy will not be limited by study design, year or type of article. The search terms will be truncated and modified to capture both American and British spelling, if required. We will include all relevant medical disciplines (ie, paediatrics, family medicine and psychiatry) because we anticipate that the articles will originate from many disciplines. We will maintain a 'wide angle' approach to generate breadth of coverage ${ }^{20}$ All study designs will be eligible for inclusion (RCT, quasi-RCT, non-RCT; quasiexperimental (Interupted Time Series (ITS) and Control Before and After (CBA) study); observational (cross-sectional, cohort, case control, case series, case report); qualitative (interview, observation, case study and survey); and mixed method (survey or interview including qualitative and quantitative data)).

To supplement the search, we will scan the reference lists of included studies for studies that might have been missed during the search process. Because our final scoping review is only as good as our literature search methodology, the search strategy for the main electronic databases (ie, MEDLINE and PsycInfo) will be peer reviewed by another experienced information specialist. This step will be conducted using the Peer Review of Electronic Search Strategies checklist. ${ }^{24}$ This additional step is necessary to ensure the literature search is valid, comprehensive and reproducible. ${ }^{21}$ Although the strengths of scoping studies include the breadth and depth of evidence covered in a given field, practical issues related to time, funding and access to resources make it difficult to determine the depth to which we should carry out the information synthesis. ${ }^{21}$

\section{Screening}

We will conduct a calibration exercise prior to the screening process to ensure reliability in selecting articles for inclusion. It will entail independent screening of a random sample of $5 \%$ of the included citations by the entire team. Eligibility criteria will be modified if low agreement is observed between reviewers. This exercise will take place at a meeting where we will seek feedback on the eligibility criteria. Subsequently, two reviewers will independently screen the remainder of the search results using predefined relevance criteria for all levels of screening. A third reviewer will meet with the team to resolve any discrepancies.

Two levels of screening will take place: a title and abstract review for articles that fit the predefined criteria and a full-text review of articles that meet those criteria. For the first-level and second-level screening, two authors (SS and GF) will independently screen the titles and abstracts of all retrieved citations. They will compare their results and resolve any disagreements. A third reviewer (PJM) will be called in if the disagreements cannot be resolved. In second-level screening, GF and SS will independently assess the full-text articles to determine whether they meet the inclusion/exclusion criteria. They will discuss and resolve any disagreements between themselves. If there is no resolution, a meeting will be held with a third reviewer (PJM) to obtain full consensus. The Preferred Reporting Items for Scoping Review checklist will be followed, and the four-phase Preferred Reporting Items for Systematic 
Table 1 Research article abstraction form

\begin{tabular}{ll} 
Extraction item & Details \\
\hline Citation &
\end{tabular}

Reviewer

Country

Aims/purpose

Setting

Theoretical approach

Sampling

Sample (participant) characteristics

Medical discipline

Stage of career

Research design

Data collection

Data analysis

Results/findings

Conclusion

Recommendations for future research

Reviews and Meta-Analyses diagram will display search results and progression of studies screened, assessed for eligibility and included in the synthesis. ${ }^{24} 25$

\section{Data abstraction}

The review team will test a draft data abstraction form on a random sample of 10 articles (see tables 1 and 2 for the data abstraction forms). The form will include study characteristics, such as authorship and year of publication; methods and methodology, including study design

\section{Table 2 Review/editorial/commentary articles abstraction form}

\begin{tabular}{ll}
\hline Extraction item & Details \\
\hline Citation &
\end{tabular}

Reviewer

Type of article

Country

\section{Setting}

\section{Purpose}

Medical discipline

Stage of career

\section{Definition of grief/bereavement/mourning}

Why is grief training important?

Themes

Paradigms/constructs that contribute to the article

Practices of grief training

Impact/outcomes (how is it adding value?)

Recommendations/future research

Conclusion and data collection methods; and the overall findings as they relate to grief training in medical education and continuing professional development. Two investigators will independently review each article and extract the relevant data. Differences in the data abstracted will be resolved through discussion or the involvement of a third reviewer. As recommended by Arksey and O'Malley, formal appraisal of the study methodology will not be conducted. ${ }^{22}$ Scoping reviews generally aim to provide a descriptive overview of the reviewed material without critically appraising individual studies. The aim of this scoping review is to identify and describe the existing literature of grief training in medical education.

Reference Manager and Excel spreadsheets will be used to store and organise the literature found in the scoping review. A Dropbox account will be created for the reviewers to share information. Dropbox is a file hosting service that provides cloud storage and file synchronisation.

\section{Synthesis}

The synthesis phase of the study will identify the key dimensions of grief training in medical education and continuing professional development. We will chart the literature by our research question. For example, we will report the number of studies by medical discipline, stage of training, pedagogical methods used and other dimensions. This approach will allow us to identify key research gaps in the literature, which will drive the development of a research agenda in this important area. It will also help us to target areas that require future systematic review. We will focus on synthesising the findings from the included studies in a manner that will not alter the intended message as originally presented in the original article. Data analysis will involve both quantitative and qualitative approaches to mapping the literature. We will provide a descriptive numerical summary and a thematic analysis. The descriptive numerical summary will include the overall number of studies included, the types of study design, publication year, characteristics of the study population and countries where the studies were conducted. The thematic analysis will resemble a qualitative content analysis to capture common themes and patterns in the data. We will use the qualitative software NVivo 10 to facilitate this process. In addition, we anticipate creating a table that lists gaps in the research evidence.

\section{DISSEMINATION AND ETHICS}

We plan to present this work at medical education conferences nationally and internationally. In addition to presenting this protocol paper, we will publish the results in a peer-reviewed academic journal. We have the support of several directors of medical education at our institution who are interested in the results, because there is a growing focus on humanism in medical education as a way of decreasing burnout among medical students, residents and faculty.

Acknowledgements The authors would like to thank Hema Žbogar for her editorial contributions to the manuscript. 
Contributors SS led the production, conceptualisation and writing of this scoping review manuscript. GF was involved in all aspects of the scoping review and participated in the manuscript preparation. RS provided feedback on the background, methodology and manuscript preparation. PJM contributed to the conceptualisation of this work and the manuscript preparation. SB developed the search strategy, conducted the search and provided feedback on the scoping review methodology. All authors give approval for the publishing of this protocol manuscript.

Funding The authors' work is supported in part by the Medical Psychiatry Alliance, a collaborative health partnership of the University of Toronto, the Centre for Addiction and Mental Health, the Hospital for Sick Children, Trillium Health Partners, the Ontario Ministry of Health and Long-Term Care and an anonymous donor.

Competing interests None declared.

Patient consent Not required.

Ethics approval This study did not require ethics review, because it only included data from publicly available materials.

Provenance and peer review Not commissioned; externally peer reviewed.

Open access This is an open access article distributed in accordance with the Creative Commons Attribution Non Commercial (CC BY-NC 4.0) license, which permits others to distribute, remix, adapt, build upon this work non-commercially, and license their derivative works on different terms, provided the original work is properly cited, appropriate credit is given, any changes made indicated, and the use is non-commercial. See: http://creativecommons.org/licenses/by-nc/4.0/.

\section{REFERENCES}

1. Kreicbergs U, Valdimarsdóttir U, Onelöv E, et al. Anxiety and depression in parents 4-9 years after the loss of a child owing to a malignancy: a population-based follow-up. Psychol Med 2004;34:1431-41.

2. Lannen PK, Wolfe J, Prigerson HG, et al. Unresolved grief in a national sample of bereaved parents: impaired mental and physical health 4 to 9 years later. J Clin Oncol 2008;26:5870-6.

3. Black D. Coping with loss. Bereavement in childhood. BMJ 1998;316:931-3.

4. Li J, Hansen D, Mortensen PB, et al. Myocardial infarction in parents who lost a child: a nationwide prospective cohort study in Denmark. Circulation 2002;106:1634-9.

5. Walker WM. Sudden cardiac death in adults: causes, incidence and interventions. Nurs Stand 2010;24:50-6.

6. American Psychiatric Association. Major depressive disorder and the 'bereavement exclusion.'. Washington DC: American Psychiatric Association Washington, 2013.
7. Bonanno GA, Keltner D. Facial expressions of emotion and the course of conjugal bereavement. J Abnorm Psychol 1997;106:126-37.

8. American Psychiatric Association. Diagnostic and statistical manual of mental disorders. 5th ed: American Psychiatric Pub, 2013.

9. Stroebe MS, Hansson RO, Stroebe WE, et al. Handbook of bereavement research: consequences, coping, and care. Washington, DC: American Psychological Association, 2001.

10. Ghesquiere A, Martinez J, Jalali C, et al. Training residents in depression and grief. Clin Teach 2018;15:114-9.

11. Rosenbaum ME, Lobas J, Ferguson K. Using reflection activities to enhance teaching about end-of-life care. J Palliat Med 2005;8:1186-95.

12. Rosenbaum ME, Ferguson KJ, Broderick A. Five-Year Experience: reflective writing in a preclinical end-of-life care curriculum. Perm J 2008;12:36

13. Schiess C, Walshe M, Wildfeuer S, et al. Undergraduate curricula in palliative medicine: a systematic analysis based on the palliative education assessment tool. J Palliat Med 2013;16:20-30.

14. O'Connor M, Breen LJ. General Practitioners' experiences of bereavement care and their educational support needs: a qualitative study. BMC Med Educ 2014;14:59.

15. Vazirani RM, Slavin SJ, Feldman JD. Longitudinal study of pediatric house officers' attitudes toward death and dying. Crit Care Med 2000;28:3740-5.

16. Tait GR, Hodges BD. End-of-life care education for psychiatric residents: attitudes, preparedness, and conceptualizations of dignity. Acad Psychiatry 2009;33:451-6.

17. Lathrop D. Disenfranchised Grief and Physician Burnout. Ann Fam Med 2017;15:375-8.

18. Redinbaugh EM, Schuerger JM, Weiss LL, et al. Health care professionals' grief: a model based on occupational style and coping. Psychooncology 2001;10:187-98.

19. Linklater GT. Educational needs of foundation doctors caring for dying patients. J R Coll Physicians Edinb 2010;40:13-18.

20. Levac D, Colquhoun H, O'Brien KK. Scoping studies: advancing the methodology. Implement Sci 2010;5:69.

21. Mays N, Roberts E, Popay J. Synthesising research evidence. In: Fulop N, Allen P, Clarke A, eds. Studying the organisation and delivery of health services: research methods. London, UK: Routledge, 2001:188-220.

22. Arksey H, O'Malley L. Scoping studies: towards a methodological framework. Int J Soc Res Methodol 2005;8:19-32.

23. Shear MK. Grief and mourning gone awry: pathway and course of complicated grief. Dialogues Clin Neurosci 2012;14:119.

24. Shamseer L, Moher D, Clarke M, et al. Preferred reporting items for systematic review and meta-analysis protocols (PRISMA-P) 2015 elaboration and explanation. BMJ 2015;349:g7647.

25. Tricco AC, Lillie E, Zarin W, et al. PRISMA extension for scoping reviews (PRISMA-ScR): checklist and explanation. Ann Intern Med 2018;169:467. 